ARTÍCULO ORIGINAL

\title{
Prevalencia de anticuerpos de tipo lgG e IgM contra COVID-19 en personal municipal esencial en Ushuaia, Tierra del Fuego, Argentina
}

\author{
Recibido: 31/10/20 Aceptado: 28/12/20
}

Adriana Basombrío ${ }^{1}$ Eva Sánchez Sciaini² ${ }^{2}$ Lucas Corradi ${ }^{3}$ José Ferro ${ }^{4}$, Gabriela Borré ${ }^{5}$.

\section{RESUMEN}

Con el propósito de ampliar el conocimiento epidemiológico sobre la circulación del SARS-CoV-2 en la ciudad de Ushuaia durante el brote ocurrido de marzo a mayo de 2020, nos planteamos realizar un estudio observacional, de corte transversal, de mayo a julio, buscando la respuesta serológica al contacto con el virus en trabajadores esenciales municipales. $\mathrm{N}=1305$. Los datos fueron ingresados a una plataforma con ingreso restringido, para luego cruzar las variables obtenidas en formato Excel. La mayoría de las personas estudiadas fueron de género masculino, con una media de edad de 38,5 años. El $20 \%$ de las personas con IgG+ tuvieron nexo epidemiológico. La prevalencia fue del $2,75 \%$. Quienes presentaron IgM+ fueron descartados por otro método, considerándose falsos positivos. La idea y realización en forma precoz del estudio fue útil para evaluar la diseminación viral asintomática en el personal municipal afectado a tareas esenciales y tomar decisiones en las primeras etapas de la pandemia. No se encontraron diferencias significativas en los grupos más expuestos. Se observaron casos de transmisión asintomática intrafamiliar. Estas pruebas serológicas no tienen fines diagnósticos ni confieren un pasaporte inmunitario. Pudimos establecer la confiabilidad de la técnica utilizada, fundamentalmente en relación a la lgG. El método se optimizó. Consideramos que las políticas públicas basadas en evidencias científicas permiten tomar mejores decisiones en beneficio de la comunidad. Creemos que el presente estudio aporta al estado de comprensión en la materia. Cada reporte puede ser de utilidad para el conocimiento de la diseminación viral.

\begin{abstract}
'Coordinación de Epidemiología, Municipalidad de Ushuaia, Tierra del Fuego, Argentina.

${ }^{2}$ Laboratorio Biosur, Tierra del Fuego, Argentina.

${ }^{3}$ Subsecretaría de de Políticas Sanitarias, Municipalidad de Ushuaia, Tierra del Fuego, Argentina.

${ }^{4}$ Hematología, Clínica San Jorge, Ushuaia, Tierra del Fuego, Argentina.

${ }^{5}$ Municipalidad de Ushuaia, Tierra del Fuego, Argentina.
\end{abstract}

Autor para correspondencia: Adriana Basombrío. Arturo Coronado 486 (9410) Ushuaia, Provincia de Tierra del Fuego, Argentina. Teléfono: (54) 2901 611525.

Email:ambasombrio@gmail.com

No existen conflictos de interés en la presente investigación.

Palabras clave: SARS-CoV-2, estudio serológico transversal, trabajadores esenciales, investigación epidemiológica. 


\section{Introducción}

En diciembre de 2019, en la ciudad de Wuhan, en la provincia de Hubei, China, se detectaron casos de neumonía de la comunidad causadas por un nuevo virus de la familia de beta coronavirus, denominado ulteriormente SARS-CoV-2.

El 30 de enero de 2020, la Organización Mundial de la Salud (OMS) declaró la emergencia de salud pública de importancia internacional en el marco del Reglamento Sanitario Internacional y declaró al síndrome respiratorio agudo severo por SARS-CoV-2 como una pandemia el 11 de marzo de 2020 (1-2). Se estableció a las personas que realizaron viajes internacionales como cumplidoras de un rol fundamental en la diseminación mundial del virus.

El 7 de enero de 2020 se identificó el genoma completo del virus.

Hasta el 30 de agosto de 2020 se han informado más de 25 millones de casos de la enfermedad en más de 210 países y territorios en el mundo (1).

El período de incubación del SARS-CoV-2 puede variar de dos a catorce días, teniendo un promedio de cinco. Los síntomas más comunes son la fiebre, la tos seca, el dolor de garganta y la dificultad para respirar, aunque se han ido agregando mayor cantidad de síntomas como la anosmia o la disgeusia, el malestar general y la astenia, entre otros. Las complicaciones pueden incluir la neumonía, el síndrome respiratorio agudo o el fallo multiorgánico; estos dos últimos como consecuencia de la cascada de activación de citoquinas (3-5).

Su mecanismo de transmisión es, generalmente y con mayor frecuencia, de persona a persona a través de las pequeñas gotas de saliva que se emiten al hablar, estornudar o toser. Se disemina, principalmente, cuando las personas están en contacto cercano, aunque también se puede difundir al tocar una superficie contaminada y luego de llevar las manos contaminadas a la cara, la boca y/o los ojos. Un factor clave en la transmisibilidad del virus es el alto nivel de desprendimiento del mismo en el tracto respiratorio superior, incluso entre pacientes presintomáticos o asintomáticos (6-12).

En un estudio realizado en la municipalidad de Vo, Italia, no se encontraron diferencias estadísticamente significativas entre personas sintomáticas y asintomáticas en la carga viral medida por equivalentes genómicos inferidos por los datos del ciclo viral (13), llegando a la conclusión que la transmisión viral puede darse a través de los pacientes sintomáticos como aquellos que nunca manifestaron síntomas.

Si bien ya se dio comienzo a la inmunización por medio de la vacuna -en este caso la Sputnik V- todavía el número de vacunados es bajo y deben completarse segundas dosis, por lo que la forma de abordaje sobre la que hay que seguir insistiendo es acerca de la comunicación de las medidas de prevención: el distanciamiento físico, la utilización de mascarilla comunitaria, el lavado frecuente de manos y la importancia de no mantener reuniones sociales. Para aquellas personas infectadas por SARS-CoV-2, la terapia sintomática y de apoyo que sea requerida según el caso. Todos los esquemas terapéuticos específicos, incluido el plasma de convaleciente, se encuentran en proceso de investigación.

Dado que el COVID-19 (denominación que se dio luego a la infecciónporSARS-CoV-2) seencuentra en permanenteestado de dinamismo, ya que lleva muy poco tiempo en el mundo, la información obtenida a través de diferentes investigaciones nos obliga a ir actualizando en forma constante nuestros conocimientos. En este caso, específicamente, nos referimos a los diferentes test serológicos.

En la ciudad de Ushuaia la epidemia se ha presentado fundamentalmente por conglomerados, habiendo sido definida la transmisión comunitaria en abril por el Ministerio de Salud de la Nación, al momento de diseñar el presente estudio, durante un breve período. La totalidad de los casos de la primera ola, comenzada el 15/03/20, ascendió a 209 casos.

Se estima que el número de personas que han tomado contacto con la infección son mayores a los que se diagnostican con el hisopado nasofaríngeo por técnica de PCR, debido a la frecuencia de formas asintomáticas o con escasos síntomas (14-16).

La mayoría de los datos serológicos disponibles de COVID-19 provienen de personas que han sido hospitalizadas con infección grave. En este grupo, alrededor del $90 \%$ desarrolla anticuerpos IgG dentro de las primeras dos semanas de infección sintomática, y este aspecto coincide con la disminución de la carga viral del individuo afectado.

De acuerdo a varios artículos publicados (17-29), los test de identificación de anticuerpos IgG e lgM no tienen una función diagnóstica y algunos puntos se encuentran en discusión. Realizar este estudio nos permite aportar al estado del conocimiento en la materia. La verdadera utilidad de estos 
tipos de pruebas se definirá a medida que los países y las localidades vayan teniendo sus propias conclusiones. De hecho, son varias las investigaciones que se están llevando a cabo en el mundo en relación a los test serológicos de búsqueda de anticuerpos contra el SARS-CoV-2.

Dada la documentación de las formas asintomáticas u oligosintomáticas de la infección por COVID-19 planteamos la realización del presente estudio de prevalencia en trabajadores esenciales municipales pudiendo a través de ello evaluar tanto casos asintomáticos en este grupo de alta exposición como la utilidad de las medidas de prevención adoptadas y la probable diseminación viral vía personal esencial.

Consideramos que esta investigación permitirá tener, entonces, una información de importancia para el mejor entendimiento epidemiológico de la pandemia en la ciudad en el período estudiado y a su vez realizar un aporte al estado del conocimiento en la materia.

\section{Materiales y métodos}

Estudio observacional, de corte transversal. Período estudiado: mayo a julio de 2020 .

\section{Objetivos del estudio}

- Ampliar el conocimiento epidemiológico sobre la circulación del virus en la ciudad, a través del testeo serológico de personal esencial.

- $\quad$ Estimar la prevalencia de Sars-CoV-2 en trabajadores esenciales asintomáticos.

Se seleccionaron a los trabajadores municipales que debieron cumplir labores esenciales durante la cuarentena.

Cabe mencionar que se presentaron dificultades en el tiempo de recepción de los kits adquiridos, lo que nos llevó a un cierto retraso en el comienzo de la implementación del estudio, así como también debimos mantener unas semanas de intervalo esperando la recepción de los nuevos envíos.

La recolección de los datos se llevó a cabo vía web a través de un sistema diseñado por el área de informática municipal, con una ficha en la cual se incluyeron las diferentes variables a ser completadas. La base de datos se obtuvo en formato Excel y se adoptó para el cruce de las variables estudiadas, utilizándose además para algunos cálculos estadísticos el SPSS.
Las variables que fueron tenidas en cuenta son las siguientes:

- $\quad$ Datos de identificación personal: nombre y apellido, DNI, fecha de nacimiento, edad, género.

- Datos de contacto: teléfono, correo electrónico, domicilio.

\section{- Comorbilidades.}

- $\quad$ Presencia de síntomas relacionados a COVID-19 en días o meses previos al estudio.

\section{- $\quad$ Cantidad de convivientes.}

Fue diseñado un consentimiento informado explicando los alcances del estudio y las características del método de testeo a realizar. Todos los voluntarios en el estudio lo firmaron. Dicho consentimiento, con la firma de la persona a quien se le practicó el test serológico para SARS-Cov-2, fue debidamente archivado.

La devolución de los resultados se realizó en forma individual a través del correo electrónico provisto por cada trabajador. Se siguió con los lineamientos éticos de la Asociación Médica Mundial de Helsinki y Tokio (30).

Proceso de extracciones: fue diseñado un cronograma para las extracciones, a partir de los listados obtenidos de los grupos de personas mencionados, en varios puntos de extracción debidamente acondicionados con todas las medidas de bioseguridad.

Principio de la prueba utilizada: COVID-19 IgG/lgM ANTIBODY RAPID TEST (Hangzhou Inmuno Biotech Co.) es una prueba rápida para la detección cualitativa in vitro de anticuerpos IgG e IgM para SARS-CoV-2 en suero, plasma, sangre entera venosa u obtenida por punción digital. Es considerada una prueba indirecta, ya que detecta la respuesta inmunológica y no directamente al virus.

Los anticuerpos que son detectados indican una respuesta inmunológica al SARS-CoV-2, ya sea con desarrollo de síntomas de la infección o en casos en los cuales la infección haya sido asintomática.

Debido a la gran variabilidad de síntomas de acuerdo a la respuesta inmunológica de cada individuo, las pruebas serológicas son más efectivas a partir de los diez a catorce días desde la sospecha de exposición. 
El dispositivo contiene una membrana de nitrocelulosa contenida en un casette plástico, el cual evita derrames y la inoculación correcta de la muestra. Posee un sector indicado como C: Control, G: Ac IgG y M: Ac IgM y una región absorbente de muestra.

Durante la etapa de diseño del estudio se llevó a cabo la validación y optimización del método. Se seleccionó la técnica de punción digital, ya que la misma presentaba varias ventajas con respecto de a los demás tipos de muestra.

La totalidad de las determinaciones fueron realizadas con el mismo lote de reactivos con el objetivo de evitar inconsistencias y que la validación del método sea válida durante todo el período de muestreo.

\section{Procedimiento optimizado seleccionado}

1. Llevar a temperatura ambiente el kit y resto de los materiales.

2. Preparar el sector de trabajo de tal manera de evitar la exposición prolongada del profesional operante y el paciente.

3. Retirar el kit del envoltorio y rotular.

4. Limpiar el dedo seleccionado con solución alcohólica al 70\%, luego de la evaporación natural se procede a

\section{Figura 1. Distribución de la muestra estudiada por género en porcentajes. $\mathrm{N}=1305$}

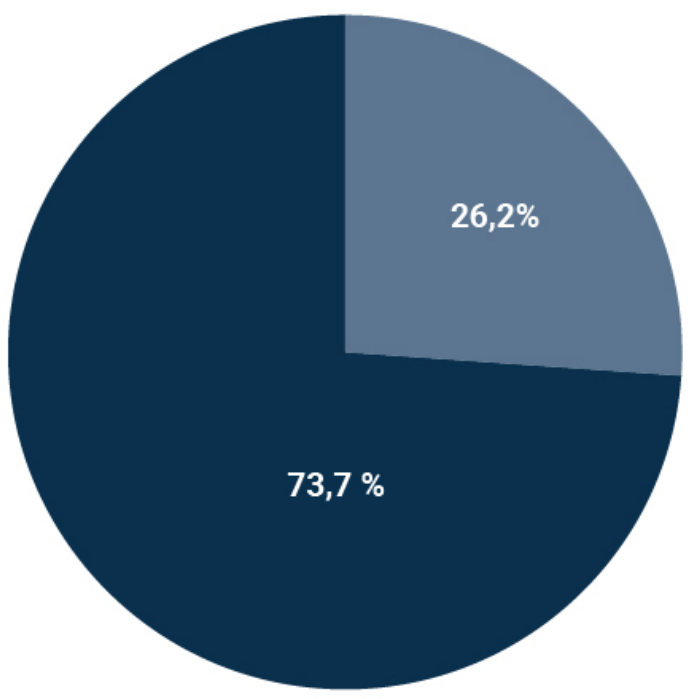

Masculino
Femenino la punción con lanceta estéril, se colecta una gota de sangre con la pipeta provista para tal fin y se coloca en la región absorbente del dispositivo de tal forma que no haya desbordes, salpicaduras ni muestra insuficiente.

5. Colocar inmediatamente tres gotas de buffer e inclinar el kit a 45 grados con el objetivo de obtener migración de la muestra por capilaridad y no por desborde, evitando lectura dificultosa.

6. Leer el resultado con luz adecuada entre los diez y quince minutos posteriores.

\section{Desempeño}

El desempeño informado por el fabricante, evaluado en pacientes sintomáticos y asintomáticos, fue el siguiente:

\begin{tabular}{|l|c|c|c|} 
& Resultado & IgG & IgM \\
\hline Infección 5 a 10 días & Sensibilidad & $70,0 \%$ & $20,0 \%$ \\
\hline Infección 11 a 28 días & Sensibilidad & $94,4 \%$ & $20,0 \%$ \\
\hline No infección & Especificidad & $>99,0 \%$ & $>99,0 \%$ \\
\hline 15 réplicas & Precisión & \multicolumn{2}{|c|}{$>99,0 \%$}
\end{tabular}

El kit utilizado se encuentra registrado en ANMAT y publicado en el listado de reactivos autorizados para COVID-19 en el marco de la emergencia sanitaria. Los mismos se encuentran organizados en las categorías Detección de COVID-19, Pruebas serológicas para COVID-19, Pruebas serológicas para COVID-19 - test rápidos y Test rápidos de Antígenos

\begin{tabular}{c|c|c|c} 
ASSERCA & $\begin{array}{c}\text { Test Rápido } \\
\text { COVID-19 } \\
\text { IgG/IgM. }\end{array}$ & $\begin{array}{c}\text { Hangzhou } \\
\text { Immuno } \\
\text { Biotech Co } \\
\text { Ltd. (China) }\end{array}$ & $\begin{array}{c}\text { Test Rápido } \\
\text { Inmunocro- } \\
\text { matográfico }\end{array}$ \\
\hline
\end{tabular}

\section{Resultados}

\section{Descripción de la muestra estudiada}

El grupo estudiado estaba integrado por trabajadores esenciales municipales, quienes continuaron sus ocupaciones, cada uno con su especificidad, desde el comienzo de la cuarentena. $\mathrm{N}=1305$.

Distribución por género: se observó una gran mayoría de varones sobre las mujeres. Por las características del tipo de trabajo municipal esencial hubo una amplia 


\section{Figura 2. Distribución de la muestra estudiada por edades, en número absolutos}

600

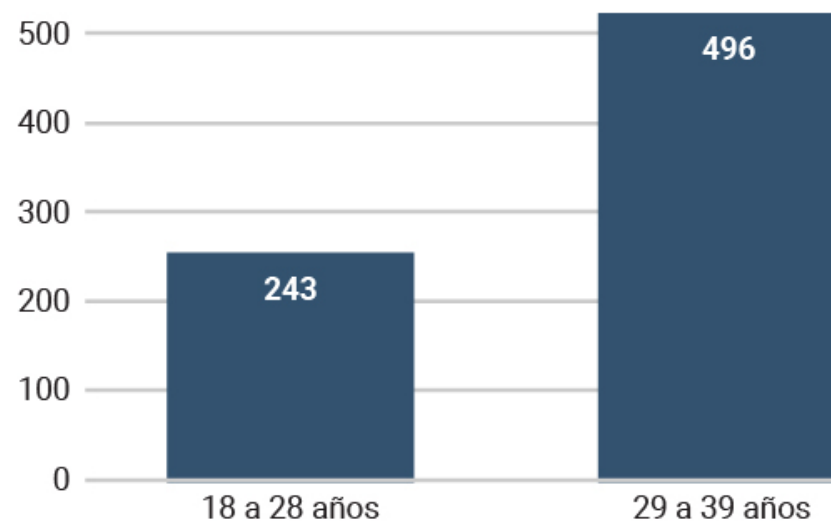

predominancia de ocupaciones que requieren de una fuerza física que en términos generales desarrollan los varones con mayor frecuencia (ejemplo: recolectores de residuos domiciliarios, transportistas, entre otros).

Distribución por edades: La mayoría de las personas se encuentran entre los 25 y los 46 años. No se observaron diferencias por edades en la distribución por género. La media de edad fue de 38,5 años.

\section{Resultados del estudio}

Se detectaron 36 personas con IgG positiva, obteniendo una prevalencia del $2,75 \%$.

Distribución por edades en el grupo de personas con IgG positiva: la mayoría de las personas se encuentran

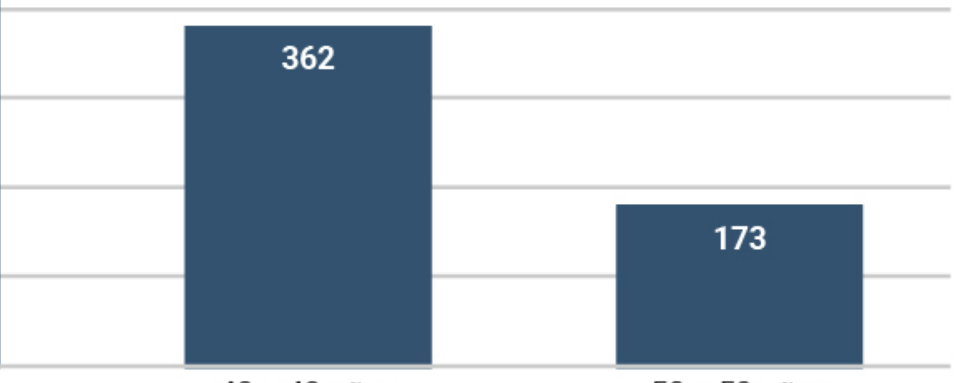

40 a 49 años
50 a 59 años

entre los 40 a 49 años, lo que corresponde al $47,2 \%$ de la muestra. La media fue de 39,5 años.

En cuanto al nexo epidemiológico, dos personas declararon haber realizado viajes al exterior durante febrero de $2020 \mathrm{y}$ una manifestó haber tenido contacto con un compañero de trabajo con diagnóstico de COVID-19. Este dato surgió de la anamnesis realizada al momento del estudio.

En la misma anamnesis dirigida en el momento del testeo, tres personas manifestaron haber tenido síntomas leves y uno moderado. Las personas con síntomas leves no consultaron, y la persona con síntomas moderados realizó consulta con realización de PCR, la cual fue negativa en dos oportunidades. Todas estas personas presentaron IgG positiva. Las personas con síntomas leves no consultaron y no realizaron aislamiento.

\section{Figura 3. Distribución por edades en el grupo de personas con lgG positiva, en números} absolutos. $\mathbf{N}=\mathbf{3 6}$

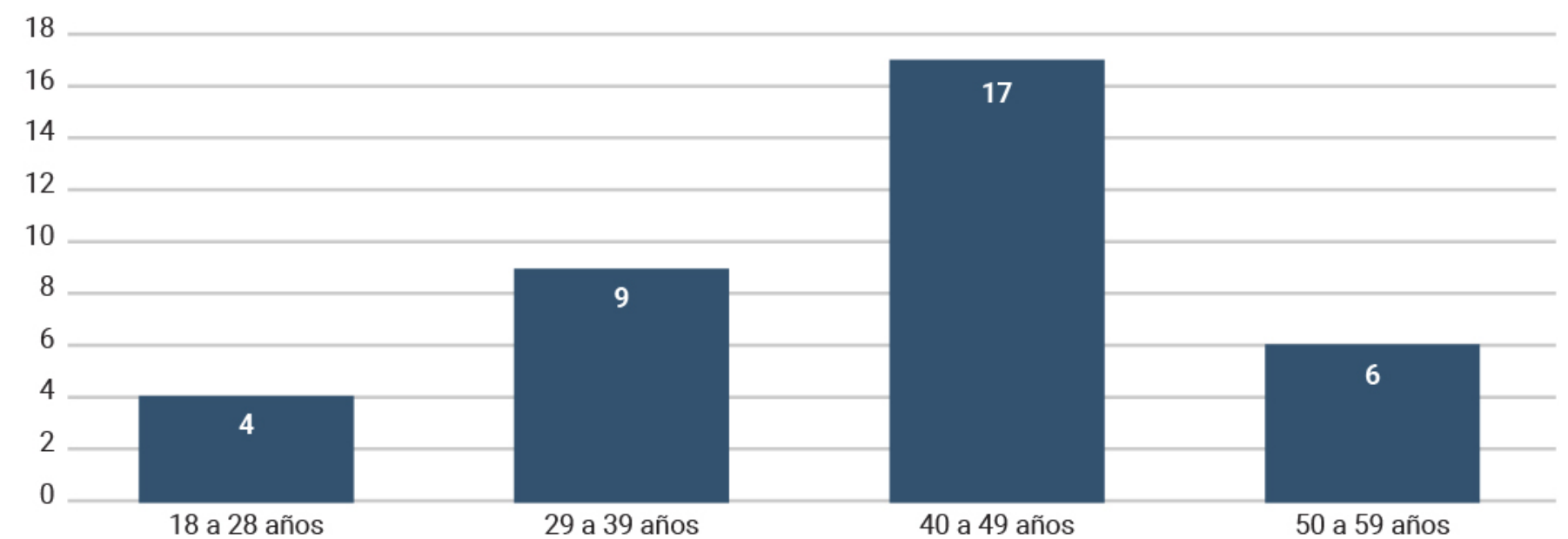

$N=1305$. Prevalencia: $2,75 \%$. 


\section{Mapa de distribución geográfica de los/las trabajadores estudiados y casos positivos}

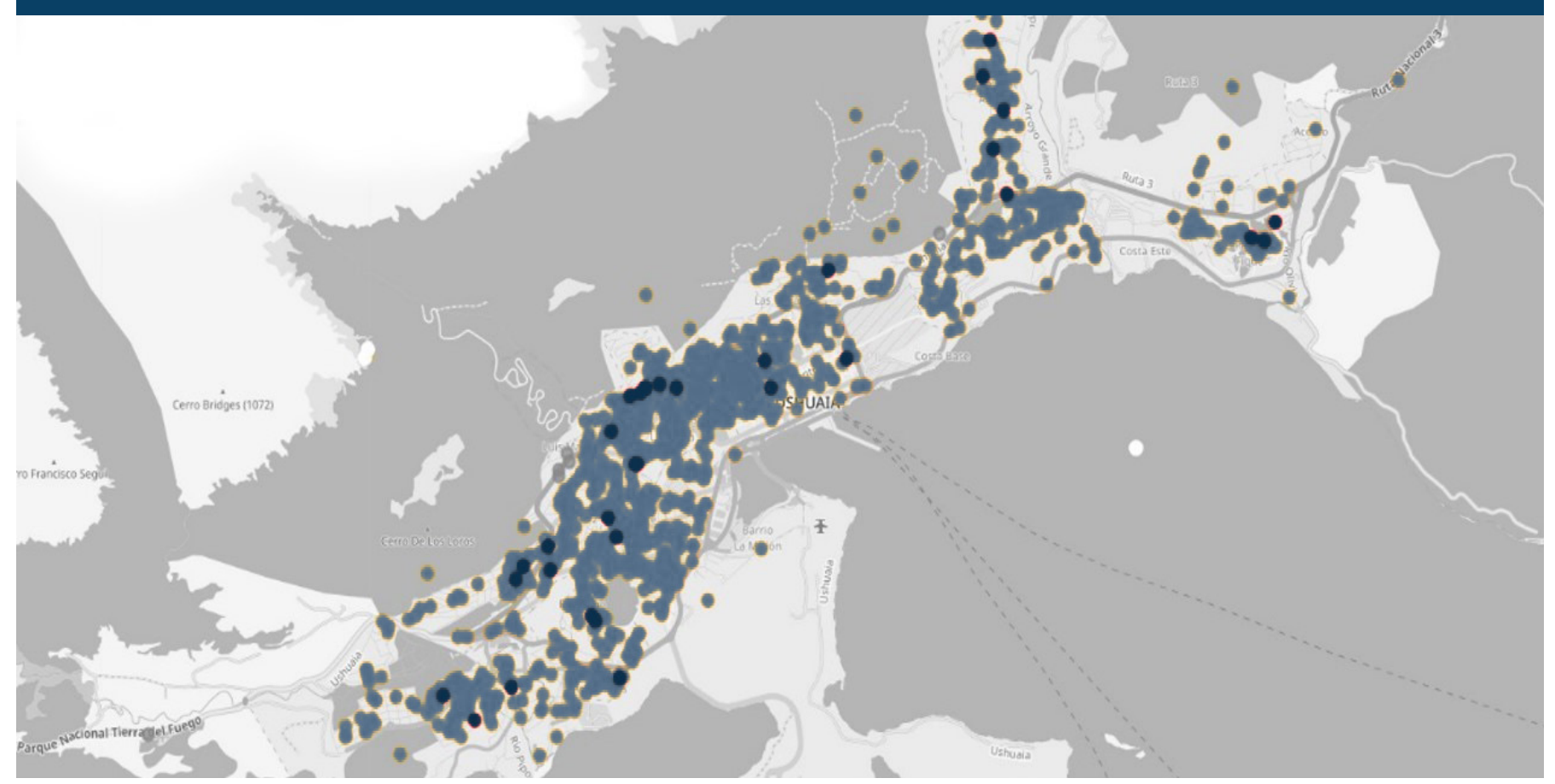

Criterio de exclusión: tener síntomas relacionados a COVID-19 al momento del test.

Encontramos cuatro personas con IgM positivas, que fueron descartadas a través de otra muestra y otra técnica.

Distribución de la residencia de las personas estudiadas y casos positivos detectados por IgG. Georreferenciamiento

En el mapa puede observarse que las viviendas de los trabajadores municipales esenciales están distribuidas a lo largo y a lo ancho de la ciudad. Asimismo, los casos positivos, marcados en negro, se encuentran distribuidos en forma casi homogénea.

\section{Discusión}

Consideramos que la idea y realización en forma precoz del estudio (teniendo en cuenta que la ciudad atravesaba su primer pico de contagios), al cual decidimos llamar de prevalencia temprana, fue de utilidad para evaluar la diseminación viral asintomática en el personal municipal afectado a tareas esenciales y poder así tomar decisiones en las primeras etapas de la pandemia. Las tasas de transmisión asintomática, las cuales varían en los distintos estudios, quedaron evidenciadas en nuestra muestra, confirmando que la tasa de casos asintomáticos es mayor a lo pensado inicialmente.
Dada la documentación de las formas asintomáticas u oligosintomáticas de la infección por COVID-19, planteamos la realización del presente estudio de prevalencia en trabajadores esenciales municipales pudiendo a través de ello evaluar casos asintomáticos y oligosintomáticos en este grupo de alta exposición, así como la utilidad de las medidas de prevención adoptadas y la probable diseminación viral vía personal esencial.

No se encontraron diferencias significativas dentro de los trabajadores esenciales municipales en los grupos con mayor exposición (por ejemplo, trabajadores de la salud vs. recolectores de residuos) como se podía suponer inicialmente.

Se observaron en nuestra muestra casos de transmisión asintomática intrafamiliar, así como contacto de convivientes que no se habrían contagiado. Esto nos obliga a pensar si esta diferencia podría deberse a que los pacientes asintomáticos podrían tener menor infectividad que aquellos con síntomas manifiestos, así como que algunos individuos no desarrollen anticuerpos contra SARS-CoV-2 o estos estén en niveles no detectables por nuestro método.

De todos modos, estos casos no fueron incluidos en el análisis de los datos.

Los resultados de estas pruebas pueden ser importantes 
en este contexto para detectar los grupos de personas que han estado infectadas, y así poder investigar probables focos de transmisión a través de procesos de georreferenciación.

Tenemos claro que estas pruebas serológicas no tienen fines diagnósticos ni confieren un pasaporte inmunitario.

Por otra parte, pudimos establecer confiabilidad de la técnica utilizada, fundamentalmente en relación a la lgG. El método se optimizó.

A raíz de los resultados podremos realizar una continuidad de este estudio evaluando a los sujetos positivos a mediano plazo para determinar si persisten con niveles detectables de anticuerpos a lo largo del tiempo. Esto nos permitiría realizar un seguimiento cercano de este grupo para evaluar la inmunidad a lo largo del tiempo y posibilidades de reinfección, debido a la elevada exposición de nuestra cohorte.

Consideramos que las políticas públicas basadas en evidencias científicas permiten tomar mejores decisiones en beneficio de la comunidad optimizando recursos.

Creemos que el presente estudio aporta en alguna medida al estado de comprensión en la materia, dentro de los estudios de este tipo que se han llevado a cabo de manera nacional e internacional. Cada reporte puede ser de utilidad para el conocimiento de la diseminación viral como así de sus formas de presentación y transmisión.

\section{Reconocimientos}

A las autoridades de la Municipalidad de Ushuaia, por comprender la importancia de llevar a cabo el presente estudio, facilitar los kits para poder realizarlo e impulsarlo.

Al equipo del Área de Informática Municipal que facilitó la base de datos, con su georreferenciamiento.

Al equipo que colaboró con la carga de datos.

Al equipo que colaboró en la implementación operativa de la toma de muestra en cada espacio de trabajo, según la necesidad y las características del grupo a estudiar. 


\section{Bibliografía}

1. Organización Mundial dela Salud. Disponible en https://www. who.int/es/emergencies/diseases/novel-coronavirus-2019

2. Li R, Pei S, Chen B, et al. Substantial undocumented infection facilitates the rapid dissemination of novel coronavirus (SARS-CoV2). Science [Internet] 2020 [accessed 2020 Apr 10]; Disponible en: https://science.sciencemag.org/content/ earle/2020/03/24/science.abb3221

3. Wu JT, Leung K, Bushman M, et al. Estimating clinical severity of COVID-19 from the transmission dynamics in Wuhan, China. Nature Medicine [Internet] 2020 [acceso 2020 Apr 8]; 1-5. Disponible en www.nature.com/articles/s41591-0200822-7

4. Jin, $Y$ y col. Hechos salientes en la inmunopatología de la infección por SARS-cov2. Viruses 2020, 12,372; doi:10.3390/ v12040372

5. Report 12 - The global impact of COVID-19 and strategies for mitigation and suppression. Imperial College London. [accessed 2020 Apr 7]; Available from: http://www.imperial. ac.uk/medicine/departments/school-public-health/ infectious-diseaseepidemiology/mrc-global-infectiousdisease-analysis/covid-19/report-12-global-impact-covid-19/

6. Gandhi, M. el al. Transmisión presintomática del SARScov2. NEJM DOI: 10.1056/NEJMe2009758 Asymptomatic Transmission, the Achilles' Heel of Current Strategies to Control Covid-19

7. Kimball A. Asymptomatic and Presymptomatic SARS-CoV-2 Infections in Residents of a LongTerm Care Skilled Nursing Facility - King County, Washington, March 2020. MMWR

8. Arons MM, Hatfield KM, Reddy SC, et al. Presymptomatic SARS-CoV-2 infections and transmission in a skilled nursing facility. N Engl J Med. DOI: 10.1056/NEJMoa2008457

9. W. Wei, Z. Li, C. Chiew y col. Fuente: MMWR Morb Mortal Wkly Rep. ePub: 1 April 2020 Presymptomatic Transmission of SARS-CoV-2 - Singapore, January 23-March 16, 2020

10. Mizumoto K, Kagaya K, Zarebski A, Chowell G. Estimating the asymptomatic proportion of coronavirus disease 2019 (COVID-19) cases on board the Diamond Princess cruise ship, Yokohama,Japan, 2020. Eurosurveillance [Internet] 2020 [accessed 2020 Apr 10];25(10):2000180. Available from: https://www.eurosurveillance.org/ content/10.2807/1560-7917.ES.2020.25.10.2000180

11. MMRWRep. 2020 [accessed 2020 Apr 10];69. Available from: https://www.cdc.gov/mmwr/volumes/69/wr/mm6913e1. htm

12. Nishiura $H$, Kobayashi T, Suzuki A, et al. Estimation of the asymptomatic ratio of novel coronavirus infections (COVID-19). Int J Infect Dis 2020; www.ncbi.nlm.nih.gov/ pubmed/32179137
13. Lavezzo, E. et al. Med Rxuvpreprprint. Supression of COVID19 outbreack in the municipality of Vo, Italy. https//:doi. org/10.1101/2020 04.17.20053157

14. TanWLuYZhang Jetal. Viral kineticsand antibody responses in patients with COVID-1 MedRxiv. 2020; (published online March 26.) (preprint) https://doi.org/10.1101/2020.03.24.2 0042382.

15. Wu F Wang A Liu M et al. Neutralizing antibody responses to SARS-CoV-2 in a COVID-19 recovered patient cohort and their implications. bioRxiv. 2020; (published online April 20.) https://doi.org/10.1101/2020.03.30.20047365

16. Adams ER Anand $R$ Andersson $M$ et al. Evaluation of antibody testing for SARS-Cov-2 using ELISA and lateral flow immunoassays. bioRxiv. 2020; (published online April 20.) (preprint) https://doi.org/10.1101/2020.04.15.20066407

17. Tan $W$ Lu $Y$ Zhang $J$ et al. Viral kinetics and antibody responses in patients with COVID-1 MedRxiv. 2020; (published online March 26.) (preprint) https://doi. org/10.1101/2020.03.24.20042382

18. Altmann, D. y col. The Lancet. DOl:https://doi.org/10.1016/ S0140-6736(20)30985-5 What policy makers need to know about COVID-19 protective immunity

19. Wu F Wang A Liu M et al. Neutralizing antibody responses to SARS-CoV-2 in a COVID-19 recovered patient cohort and their implications. bioRxiv. 2020; (published online April

20. Stowell, et al. Role of serology in the Covid19 pandemic. Clinical Infectious Diseases, ciaa510, https://doi.org/10.1093/ cid/ciaa510

21. Abbasi, J. Promesas y riesgos de las pruebas de anticuerpos para COVID-19. JAMA The Promise and Peril of Antibody Testing for COVID-19

22. OMS. Population-based age-stratified seroepidemiological investigation protocol for COVID-19 virus infection. Available from: https://www.who.int/publicationsdetail/populationbased-age-stratified-seroepidemiological-investigationprotocol-for-covid-19-virusinfection

23. Terra,Pycol.Bloodtestsshow $14 \%$ of peoplearenowimmune to covid-19 in one town in Germany [Internet]. MIT Technology Review. Available from: https://www.technologyreview. com/2020/00/09/.

24. San Miguel County suspends, then recommits to its universal coronavirus testing experiment over 3day span. The Colorado Sun. Available from:https://coloradosun.com/2020/04/09/ san-miguel-county-telluride-universal-coronavirus-testing/

25. Chang, Min et al. Interpreting the COVID-19 Test Results. A Guide for Physiatrists. American Journal of Physical Medicine \& Rehabilitation: July 2020 - Volume 99 - Issue 7 p 583-585. doi: 10.1097/PHM.0000000000001471

26. Bryant J. E. et al. Serology for SARS-CoV-2: Apprehensions, opportunities, and the path forward Developing a National 
Strategy for Serology (Antibody Testing) in the United States. Gigi Gronvall, PhD et al. Johns Hopkings University. April 2, 2020

27. Sundararaj Stanleyraj Jeremiah, MD. Interpreting Diagnostic Tests for SARS-CoV-2., Department of Microbiology and Molecular Biodefense Research, Yokohama City University School of Medicine, Sethuraman, Nandini; Jeremiah, Sundararaj Stanleyraj; Ryo, Akihide.JAMA ; 323(22): 2249-2251, 2020 Jun 09.Article in English | MEDLINE | ID: covidwho-186473

28. The Laboratory Diagnosis of COVID-19- Frequently-Asked Questions. Ferric C. Fang et al. Departments of Laboratory Medicine and Microbiology and Medicine University of Washington School of Medicine Seattle, Washington, USA 98195. Published by Oxford University Press for the Infectious Diseases Society of America.30/06/20

29. Novel Coronavirus Press Archives-Public Health Department - County of Santa Clara. Available from: https://www.sccgov. org/sites/phd/Diseaselnformation/novelcoronavirus/ Pages/archives.aspx(30)

30. Declaración de Helsinski. Disponible en https://www.wma. net/es/policies-post/declaracion-de-helsinki-de-la-ammprincipios-eticos-para-las-investigaciones-medicas-en-sereshumanos/ 
Prevalence of IgG e IgM antibodies against COVID-19 in essential municipal personnel. Ushuaia, T. del Fuego, Argentina

With the purpose of expanding epidemiological knowledge about the circulation of SARS-CoV-2 in the city of Ushuaia during the outbreak that occurred from March to May of this current, we plan to carry out an observational, cross-sectional study from May to July, seeking the serological response to contact with the virus in essential municipal workers. $\mathrm{N}=$ 1316. The data were entered into a platform with restricted entry, to later cross the variables obtained in Excel format. Most of the people studied were male, with a mean age of 38 years. $20 \%$ of the people with IgG + had an epidemiological link. The prevalence was $2.88 \%$. Those who presented IgM+ were discarded by another method, being considered false positives. The early idea and implementation of the study was useful to evaluate asymptomatic viral shedding in municipal personnel affected by essential tasks and to make decisions in the early stages of the pandemic. No significant differences were found in the most exposed groups. Cases of asymptomatic interfamily transmission were observed. These serological tests do not have diagnostic purposes nor do they confer an immune passport. We were able to establish the reliability of the technique used, mainly in relation to IgG. The method was optimized. We believe that public policies based on scientific evidence allow us to make better decisions for the benefit of the community. We believe that the present study contributes to the state of understanding in the matter. Each report can be useful for understanding viral shedding.

Keywords: SARS-CoV-2, cross-sectional serological study, essential workers, epidemiological investigation. 